\title{
ANÁLISE DA COBERTURA E RESULTADOS DO EXAME PAPANICOLAOU EM UMA UNIDADE BÁSICA DE SAÚDE
}

\section{ANALYSIS OF COVERAGE AND RESULTS OF PAP SMEAR TEST IN A BASIC HEALTH UNIT}

\section{ANÁLISIS DE LA COBERTURA Y LOS RESULTADOS DE LAS PRUEBAS DE PAPANICOLAOU EN UNA UNIDAD BÁSICA DE SALUD}

\author{
Jéssica Fracalossi Feijó ${ }^{1}$, Gustavo Potratz Gonçalves ${ }^{2}$, Thaís Fonseca Chagas ${ }^{3}$, Carolina Airão Destefani ${ }^{4}$, Laryssa Caroline \\ Reis Silva ${ }^{5}$, Luísa Gonçalves Dutra de Oliveira ${ }^{6}$
}

\section{RESUMO}

O diagnóstico precoce do câncer de colo de útero é de extrema importância para redução da morbimortalidade provocada por esse tipo de neoplasia. Isso justifica um estudo que analise a cobertura e os resultados do exame Papanicolaou na população usuária do SUS, levando em conta o padrão das alterações e o tratamento proposto, bem como a proporção de mulheres que não comparece para receber o resultado do exame. Com esses objetivos, foi desenvolvida uma pesquisa quantitativa, descritiva e exploratória, utilizando-se os registros de uma Unidade Básica de Saúde (UBS) da região metropolitana do Rio de Janeiro e bancos de dados (SISCOLO e IBGE) referentes ao ano de 2011. Os resultados apontaram cobertura total de $11,25 \%$, resultados alterados em $78,66 \%$ dos casos, proporção de $29,90 \%$ de exames não entregues, tempo médio entre coleta e chegada do resultado do exame à UBS de um mês e 26 dias e tempo médio entre coleta e entrega do resultado à usuária de quatro meses e seis dias.

Descritores: Cobertura de serviços de saúde, Acesso aos Serviços de Saúde, Esfregaço Vaginal, Saúde da Mulher.

\footnotetext{
1 Graduanda da Faculdade de Medicina da Universidade Federal Fluminense, UFF, Niterói-RJ. E-mail: jessicafracalossi@id.uff.br

${ }^{2}$ Graduando da Faculdade de Medicina da Universidade Federal Fluminense, UFF, Niterói-RJ. E-mail: ; gustavo-gpg@hotmail.com

${ }^{3}$ Graduanda da Faculdade de Medicina da Universidade Federal Fluminense, UFF, Niterói-RJ. E-mail: thaisf.chagas@hotmail.com

4 Graduanda da Faculdade de Medicina da Universidade Federal Fluminense, UFF, Niterói-RJ. E-mail: carol_destefani@hotmail.com

5 Graduanda da Faculdade de Medicina da Universidade Federal Fluminense, UFF, Niterói-RJ. E-mail: aryssa_crs@hotmail.com

${ }^{6}$ Doutoranda em Saúde Pública na Escola Nacional de Saúde Pública/Fiocruz. Enfermeira do Instituto de Saúde da Comunidade/UFF. E-mail: luisa.dutra@gmail.com
} 


\begin{abstract}
Early diagnosis of uterine cervical cancer is extremely important to reduce morbidity and mortality caused by this type of cancer. This justifies a study to examine the coverage and results of the Pap smear test in SUS users, taking into account the alterations pattern and proposed treatment, and the women proportion who do not return to receive the results of such tests. With these aims, it was developed a quantitative, descriptive and exploratory research, using the records of a Basic Health Unit (UBS) in the metropolitan region of Rio de Janeiro and databases (SISCOLO and IBGE) of 2011. The results pointed total coverage of $11.25 \%$, abnormal results in $78.66 \%$ of cases, proportion of $29.9 \%$ of exams undeliverable, average time between collection and arrival of the exam result into UBS of one month and 26 days and average time between collection and delivery of the results to the user of four months and six days.
\end{abstract}

Key words: Health Services Coverage, Health Service Accessibility, Vaginal Smears, Women's Health.

\title{
RESUMEN
}

El diagnóstico precoz del cáncer del cuello uterino es extremadamente importante para reducir la morbilidad y la mortalidad causadas por este tipo de cáncer. Esto justifica un estudio para examinar la cobertura y los resultados de la prueba de Papanicolaou en la populación usuaria del SUS, teniendo en cuenta las alteraciones y el tratamiento propuesto, así como la proporción de mujeres que no vinieron a recibir los resultados del examen. Con estos objetivos, hemos desarrollado una investigación cuantitativa, descriptiva y exploratoria, haciendo uso de los registros de una Unidad Básica de Salud (UBS) en la región metropolitana del Río de Janeiro y bases de datos (SISCOLO y IBGE) relativo al año 2011. Los resultados mostraron una cobertura total de $11,25 \%$, resultados anormales en $78,66 \%$ de los casos, proporción del 29,90\% de los exámenes que no fueron entregados, el tiempo medio entre la recolección y la llegada del resultado del examen a UBS es de uno mes y 26 días y el tiempo promedio entre la recogida y la entrega de los resultados al usuario, de cuatro meses y seis días. 
Descriptores: Cobertura de los Servicios de Salud, Accesibilidad a lós Servicios de Salud, Frontis Vaginal, Salud de La Mujer.

\section{INTRODUÇÃO}

O câncer do colo do útero é a segunda neoplasia maligna mais frequente entre as mulheres, atrás apenas do câncer de mama, sendo a quarta causa de morte de mulheres por câncer no Brasil e representando $10 \%$ de todos os tumores malignos que acometem as mulheres ${ }^{(1)}$. De acordo com dados de 2011 do Instituto Nacional do Câncer (INCA), a cada ano o câncer do colo do útero é responsável pela morte de cerca de 4.800 mulheres no Brasil e por 18.430 novos casos registrados ${ }^{(2)}$.

O câncer do colo do útero é uma neoplasia de progressão lenta, com início a partir de uma lesão pré-invasiva, passível de cura na absoluta maioria dos casos, e que progride, normalmente, durante 10 a 20 anos até atingir o estágio invasor - etapa em que a cura se torna mais difícil ${ }^{(3)}$. Essa característica explica porque o câncer do colo do útero é uma das raras moléstias malignas curáveis em $100 \%$ dos casos, quando diagnosticada precocemente ${ }^{(4)}$. O método de rastreamento do câncer do colo do útero empregado no Brasil é o exame citopatológico, também conhecido como exame Papanicolaou ${ }^{(5)}$.

O pico de incidência das lesões precursoras que progridem para carcinoma invasivo, se não detectadas e tratadas em tempo hábil, ocorre na faixa etária de 35 a 49 anos de idade ${ }^{(6)}$. O Ministério da Saúde/INCA preconiza que o exame Papanicolaou seja realizado uma vez por ano em mulheres na faixa etária de 25 a 64 anos, podendo ser realizado uma vez a cada três anos, caso haja dois exames anuais consecutivos com resultados negativos. O início da coleta deve ser aos 25 anos, apenas para as mulheres que já tiveram atividade sexual e deve seguir até os 64 anos. Após esta idade, os exames devem ser interrompidos quando apresentarem ao menos dois resultados negativos consecutivos nos últimos cinco anos. Para as mulheres com mais de 64 anos que nunca realizaram o exame Papanicolaou, deve-se realizar dois exames com intervalo de um a três anos. Se ambos forem negativos, essas mulheres podem ser dispensadas de exames adicionais ${ }^{(5)}$. A priorização desta faixa etária como população alvo justifica-se por ser a de maior ocorrência das lesões de alto grau, passíveis de serem tratadas efetivamente para não evoluírem para o câncer. Segundo a Organização Mundial de Saúde (OMS), a incidência deste câncer atinge seu pico entre os 50 e 60 anos de idade ${ }^{(7)}$. Antes dos

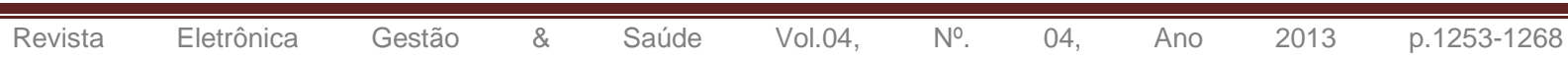


25 anos, entretanto, prevalecem lesões de baixo grau, que regredirão espontaneamente na maioria dos casos e, portanto, podem ser acompanhadas conforme recomendações clínicas. Após os 65 anos, por outro lado, se a mulher tiver feito os exames preventivos regularmente, e obtido resultados normais, o risco de desenvolvimento do câncer do colo do útero é reduzido, devido à sua lenta evolução ${ }^{(5)}$. Portanto, inicia-se o rastreamento do câncer do colo do útero na idade em que as lesões precursoras aparecem com mais frequência (a partir dos 25 anos) e mantém-se o rastreamento até a idade na qual as chances de desenvolver um carcinoma invasivo posteriormente são pequenas (até os 64 anos).

A ocorrência do câncer do colo do útero está diretamente vinculada às condições socioeconômicas do país, sendo que quase $80 \%$ dos novos casos ocorrem em países com grande desigualdade socioeconômica, ainda que o exame Papanicolaou seja tecnicamente simples e de baixo custo ${ }^{(4)}$. Segundo a OMS, com a implantação de qualidade do rastreamento citológico, uma cobertura da população-alvo de, no mínimo, $80 \%$ e a garantia de diagnóstico e tratamento adequados dos casos alterados, é possível reduzir a incidência do câncer do colo do útero invasivo em 60 a $90 \%$, em média ${ }^{(8)}$. Isso mostra que utilizando este exame como rastreador de câncer de colo uterino em programas bem estruturados, nota-se uma queda significativa das taxas de incidência e de mortalidade ${ }^{(4)}$. De fato, a experiência de alguns países desenvolvidos mostra que a incidência do câncer do colo do útero foi reduzida em torno de $80 \%$ onde o rastreamento citológico foi implantado com qualidade, cobertura, tratamento e seguimento adequado das mulheres ${ }^{(7)}$.

Embora a importância desse exame seja conhecida, vários estudos mostram que a falta de adesão ao exame Papanicolaou pela população feminina deve-se a fatores como o desconhecimento do próprio corpo, o desconhecimento do exame e da sua realização, a dificuldade de acesso, e outros fatores de ordem pessoal ${ }^{(9)}$. Isso permite supor que, a dificuldade de adesão da mulher à realização do exame pode estar relacionada ao fato de se tratar de um procedimento que requer a exposição e a manipulação da genitália feminina ${ }^{(4)}$.

A dificuldade de acesso é um fator importante que provoca a falta de adesão das mulheres ao exame Papanicolaou. $\mathrm{O}$ acesso é considerado um dos elementos fundamentais na atenção à saúde e corresponde à sua capacidade de produzir serviços e de responder às necessidades de saúde de uma determinada população. Desta forma, o conceito de acesso abrange aspectos referentes aos recursos e à organização dos serviços, os quais podem facilitar ou limitar seu uso por potenciais usuários ${ }^{(10)}$. Entretanto, acessibilidade é mais 
abrangente do que a mera disponibilidade de recursos em um determinado momento e lugar. Refere-se às características dos serviços e dos recursos de saúde que permitam seu pleno uso pela população ${ }^{(11)}$. Logo, deve-se adaptar o serviço às necessidades da população, quanto à acessibilidade e ao acolhimento. Percebe-se, desse modo, que a prestação de atendimento à população feminina necessita estar sempre sob reavaliação e reorganização, de acordo com a análise do desempenho alcançado, a fim de criar um ambiente acolhedor e de fácil acesso, que propicie a resolução do problema da falta de adesão das mulheres ao exame Papanicolaou $^{(10)}$.

O Sistema Único de Saúde (SUS) reportou, em 2009, uma cobertura de exame Papanicolaou de $18 \%$ para a população feminina na faixa etária de 25 a $59 \operatorname{anos}^{(12)}$, ou seja, foram realizados 18 exames para cada 100 mulheres da população alvo. Isto mostra uma realidade ainda distante daquela observada em países desenvolvidos, e distante da própria meta do Ministério da Saúde para o biênio 2010-2011, que foi de uma cobertura de $30 \%{ }^{(12)}$. Essa realidade evidencia a necessidade de um rastreamento mais efetivo do câncer do colo do útero e justifica um estudo que analise a cobertura e os resultados do exame Papanicolaou na população usuária do SUS.

Neste trabalho, analisou-se a cobertura de exames Papanicolaou em um bairro da região metropolitana do Rio de Janeiro, bem como os resultados dos exames realizados em uma Unidade Básica de Saúde (UBS) deste bairro.

\section{OBJETIVOS}

Este estudo teve dois objetivos gerais: analisar a cobertura total de exames Papanicolaou em um bairro da Região Metropolitana do Rio de Janeiro (RJ) e avaliar os resultados dos exames coletados no ano de 2011 em uma Unidade Básica de Saúde (UBS) deste bairro. Além destes, os objetivos específicos deste estudo foram: analisar a presença e os tipos de alteração encontrados nos resultados dos exames, verificar o acompanhamento e tratamento das usuárias, e identificar a proporção de mulheres que não retornaram para receber o resultado dos exames. Os resultados deste estudo podem servir de subsídio para a elaboração de ações de planejamento e gestão em saúde pública, voltadas para a realidade local.

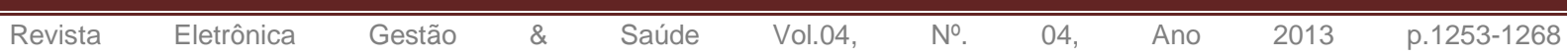




\section{MÉTODOS}

A pesquisa realizada neste trabalho teve caráter quantitativo, descritivo e exploratório. Os métodos utilizados foram a análise de dados oficiais publicamente disponíveis e o levantamento de dados secundários colhidos em registros e documentos da própria Unidade Básica de Saúde (UBS). O universo da pesquisa foram mulheres que realizaram exame Papanicolaou na UBS durante ano de 2011.

O bairro estudado possui, além da UBS, três Unidades de Saúde da Família (USF), que também foram analisadas quanto à cobertura do exame. As fontes de dados para coleta de informações sobre a população feminina do bairro e o número de exames Papanicolaou realizados pela rede pública de saúde do mesmo foram o Censo 2010, do Instituto Brasileiro de Geografia e Estatística (IBGE), e o Sistema de Informação do Câncer do Colo do Útero (SISCOLO), disponível no Banco de Dados do Sistema Único de Saúde (DATASUS). As fontes utilizadas para coleta de dados secundários, na UBS, foram: o Livro Controle, com dados de todas as usuárias que realizaram o exame na Unidade; o Arquivo de Exames, a partir do qual foi possível verificar os resultados que não foram entregues às usuárias; a Planilha Controle, preenchida apenas com dados das usuárias que apresentaram algum tipo de alteração no exame; e os prontuários disponíveis.

O Livro Controle faz parte da rotina da pré-consulta ginecológica. Ele é preenchido pelo técnico de enfermagem e contém informações sobre todas as usuárias que tiveram amostra colhida para exame, independente se a análise da amostra foi realizada no laboratório vinculado à UBS ou em outro de escolha da paciente. Para este estudo, utilizaram-se apenas dados das pacientes cujos exames foram realizados no laboratório vinculado à UBS. O Arquivo de Exames armazena os resultados de exames que ainda não foram retirados pelas usuárias. Essa fonte de dados foi utilizada para conferir se os resultados haviam sido entregues ou não. Neste estudo, os exames caracterizados como não entregues às usuárias estavam arquivados por, no mínimo, cinco meses. A Planilha Controle, contendo dados das usuárias que apresentaram resultados alterados no exame, foi desenvolvida pelo setor de Vigilância em Saúde da UBS. O objetivo desta planilha é controlar a entrega dos resultados de exames com alteração e realizar busca ativa das usuárias com alterações importantes no exame, através de telefonemas ou aerogramas, caso seja necessário. Os prontuários das pacientes foram utilizados para coletar informações sobre os resultados dos exames, quando

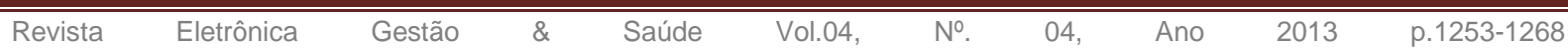


essa informação não constava nas outras fontes, e sobre a conduta médica. Além disso, os prontuários foram importantes para conferência de dados divergentes ou não preenchidos nas outras fontes.

Todos os dados secundários foram coletados durante o desenvolvimento de atividades de campo do curso de Medicina, envolvendo a participação de graduandos na rede de Atenção Básica. Estes dados foram consolidados em uma nova planilha eletrônica (Excel) e posteriormente analisados, permitindo, assim, a obtenção dos resultados que serão descritos a seguir. No total, foram analisados 1111 exames, sendo 525 pertencentes à UBS, onde cada exame realizado correspondeu a uma única usuária, e os demais 586 pertencentes às USF, onde não foi possível determinar a correspondência entre exames e usuárias.

\section{RESULTADOS}

A cobertura total realizada, em 2011, pela rede pública do bairro estudado foi de 11,25\%, sendo que a cobertura total efetiva da Unidade Básica de Saúde (UBS) foi de 5,40\% e a das três Unidades de Saúde da Família (USF) foi de 5,85\% (as coberturas efetivas da UBS e das USF consideraram apenas os seus respectivos percentuais do total de exames realizados). Para a população feminina na faixa etária de 35 a 49 anos a cobertura realizada no bairro foi de $15,46 \%$, sendo que a cobertura efetiva da UBS foi de $6,72 \%$ e a das USF foi de $8,74 \%$. Por fim, para a população de usuárias na faixa etária de 25 a 64 anos, a cobertura encontrada foi de $14,52 \%$, sendo que a cobertura efetiva da UBS foi de $6,31 \%$ e a das USF foi de $8,21 \%$. Esses resultados estão aquém da meta do Ministério da Saúde para o biênio 20102011 de uma cobertura de $30 \%$, e demonstram uma proximidade da cobertura efetiva da UBS com a das três USF.

A análise da entrega dos resultados às usuárias que realizaram exames na UBS é mostrada no gráfico da Erro! Fonte de referência não encontrada.. Observou-se que $54,86 \%$ das usuárias receberam os resultados dos exames e 29,90\% não retornaram para buscar o exame. Em 15,24\% dos casos, não foi possível constatar se os resultados dos exames foram entregues ou não, pois não havia registro de data de entrega do resultado, ainda que os

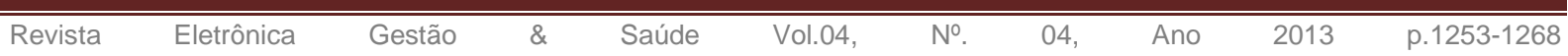


resultados não estivessem no Arquivo de Exames. Esses resultados foram classificados como "Não Encontrado".

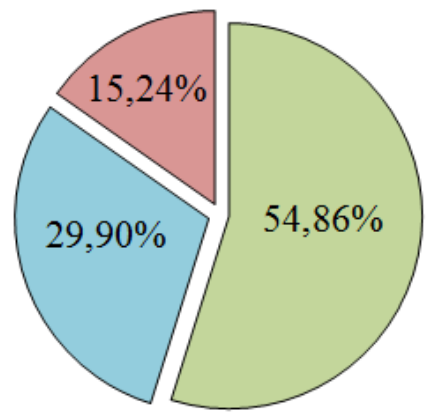

$\square$ Entregue

$\square$ Não Entregue

$\square$ Não Encontrado

Figura 1. Proporção entre resultados de exames Papanicolaou entregues, não entregues e não encontrados. UBS, 2011.

A análise da proporção de resultados de exames com ou sem alteração é mostrada no gráfico da Figura 2. Conforme é possível verificar, 78,66\% apresentaram algum tipo de alteração e $12,76 \%$ apresentaram resultados sem alteração. Em 8,57\% dos casos não foi possível constatar se os resultados dos exames estavam alterados ou não, pois não havia registro dessa informação. Esses resultados foram classificados como "Sem Informação".

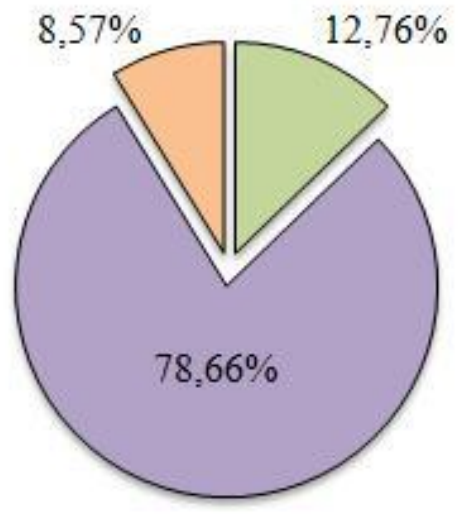

$\square$ Sem Alteração

$\square$ Alterado

$\square$ Sem Informação

Figura 2. Proporção entre resultados de exames preventivos sem alteração, alterados e sem informação. UBS, 2011.

$\mathrm{Na}$ análise dessa variável, cabe ressaltar que, dentre os resultados não entregues, havia um único caso de Lesão Intraepitelial escamosa de baixo grau (LSIL) - cujo resultado já havia sido entregue à UBS, e, mesmo havendo busca ativa, a usuária não retornou nos meses seguintes para buscar seu resultado.

Os tipos de alterações registrados nas fontes de coleta de dados foram diversos, e não havia uma uniformidade quanto à classificação utilizada para avaliar os exames. Portanto,

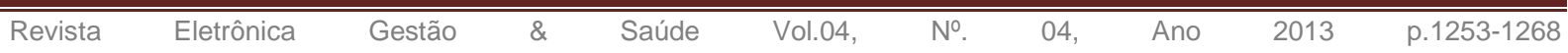


optou-se, no escopo deste estudo, por adequar os tipos de alterações, descritos nos registros disponíveis, à Classificação Citológica Brasileira de 2006.

Encontraram-se dois tipos de classificações diferentes nas fontes de coleta de dados. Primeiro, na Planilha Controle e no Arquivo de Exames, os resultados estavam descritos de acordo com as características das lâminas analisadas na citopatologia. Já nos Prontuários, os resultados estavam classificados de acordo com a Classificação Citológica de Papanicolaou de 1941, sendo estes classificados como Classe I a V. Para realizar a correlação entre as classificações acima descritas e a Classificação Citológica Brasileira de 2006, preconizada pelo Ministério da Saúde (MS) como classificação a ser utilizada no âmbito nacional, buscouse informações da Nomenclatura Brasileira para Laudos Cervicais e Condutas Preconizadas do $\mathrm{MS}^{(13)}$ e nas Diretrizes Brasileiras para Rastreamento do Câncer de Colo de Útero do INCA $^{(5)}$. Com isso, foi possível reclassificar os resultados de acordo com as descrições a seguir. Primeiramente, os exames sem alterações celulares ou classificados como Classe I foram reclassificados como "Normal". Em seguida, características descritas no laudo citopatológico como inflamação, atrofia, citólise, infiltrado inflamatório, exsudato leucocitário, ceratose, alterações reativas, núcleos ativados, e resultados classificados como Classe II foram reclassificados como "Alterações celulares benignas". Resultados com presença de células escamosas atípicas foram reclassificados como "ASCUS - Atipia celular escamosa de significado indeterminado". Por fim, os resultados classificados como Neoplasia Intraepitelial Cervical de grau I (NIC I) ou Classe III foram reclassificados como "Lesão Intraepitelial escamosa de baixo grau (LSIL)". Vale ressaltar que não apareceram outros tipos de resultados, não havendo, portanto, a necessidade de realizar as correlações para todos os possíveis tipos de alteração.

O resultado obtido nesta análise está ilustrado no gráfico da Figura 3. Do total de $78,66 \%$ de resultados alterados, 77,52\% eram compatíveis com alterações celulares benignas, apenas três casos foram compatíveis com ASCUS - Atipia celular escamosa de significado indeterminado - e outros três casos com Lesão Intraepitelial escamosa de baixo grau (LSIL), com cada conjunto de casos representando $0,57 \%$ do total de exames. Observa-se, portanto, a predominância de alterações celulares benignas, consideradas como resultado não patológico.

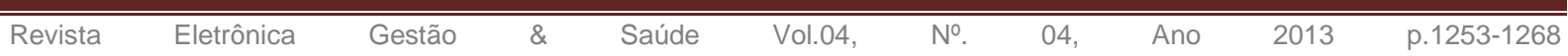




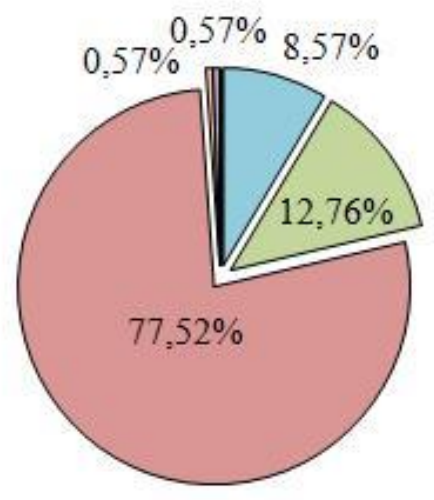

口Sem informação

$\square$ Normal

$\square$ Alterações celulares benignas

$\square$ ASCUS - Atipia celular escamosa de significado indeterminado

口Lesão Intraepitelial escamosa de baixo grau (LSIL)

Figura 3. Classificação dos registros dos tipos de alterações de acordo com a nomenclatura do MS/INCA. UBS, 2011.

A análise relativa às condutas realizadas frente aos resultados dos exames entregues é ilustrada no gráfico da Figura 4. Conforme podemos verificar, 49,31\% das usuárias receberam conduta específica, o que inclui medicação específica na maior parte dos casos, além de orientação e repetição do exame para controle. O registro destas duas últimas ações foi encontrado em uma pequena parcela dos casos estudados. Por outro lado, 22,22\% não receberam conduta específica, independente de haver alteração no resultado do exame. Em $28,47 \%$ dos casos não foi possível identificar a conduta do médico frente ao resultado do exame, seja por anotação ilegível no prontuário $(6,25 \%$ dos casos), por motivo do prontuário não ter sido encontrado $(13,54 \%$ dos casos) ou porque não havia anotação no prontuário $(8,68 \%$ dos casos $)$.

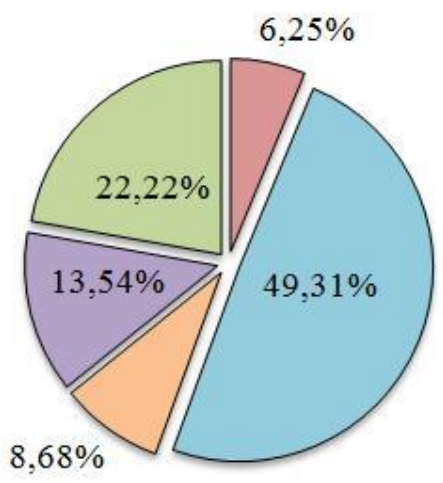

\author{
$\square$ Anotação Ilegível \\ $\square$ Conduta Específica \\ $\square$ Sem Anotação no Prontuário \\ $\square$ Prontuário não Encontrado \\ $\square$ Sem Conduta Específica
}

Figura 4. Conduta médica frente aos resultados dos exames Papanicolaou. UBS, 2011.

Os resultados da análise dos intervalos de tempo entre as etapas de coleta do material para exame, de chegada do resultado à UBS e de entrega do resultado à usuária são 
apresentados na Tabela 1, que está organizada conforme a seguir. Nas linhas da tabela são relacionados os três intervalos de tempo que foram analisados, enquanto os tempos médios e máximos de cada intervalo são apresentados nas suas respectivas colunas. Para a apresentação destes resultados em números de meses e dias considerou-se um mês como equivalente a 30 dias. Encontrou-se uma média de um mês e 26 dias entre a realização da coleta do exame e a chegada do resultado à UBS, com um tempo máximo de sete meses e 16 dias. A média encontrada para o intervalo entre a chegada do exame à UBS e entrega do exame à usuária foi de dois meses e 12 dias, com um tempo máximo de 11 meses e 4 dias. Dessa forma, a média de tempo total entre realização da coleta do exame e o recebimento do seu resultado foi de quatro meses e seis dias, com um tempo máximo de um ano, dois meses e 14 dias. Vale ressaltar que os tempos médios calculados para os intervalos entre chegada do resultado e entrega deste à usuária e entre coleta do exame e entrega deste à usuária são referentes apenas àqueles resultados de exames que foram retirados pelas usuárias e que possuíam todas as datas preenchidas adequadamente, desconsiderando-se todos os outros casos. Desta forma, é plausível admitir que a medida apresentada por estes cálculos possa estar subdimensionada.

\begin{tabular}{ll|l|l}
\multicolumn{2}{c}{ Intervalo de Tempo } & \multicolumn{1}{c}{ Médio } & Máximo \\
\hline & Entre coleta do exame e chegada do resultado & 1 mês e 26 dias & 7 meses e 16 dias \\
Entre chegada do resultado e entrega à usuária & 2 meses e 12 dias & 11 meses e 4 dias \\
Entre coleta do exame e entrega do resultado à & 4 meses e 6 dias & 1 ano 2 meses e 14 dias \\
usuária & & & \\
\hline
\end{tabular}

Tabela 1. Análise dos intervalos de tempo entre as etapas de coleta, resultado e entrega do exame Papanicolaou. Fonte: UBS, 2011.

\section{DISCUSSÃO}

Um inquérito domiciliar realizado pelo Ministério da Saúde (MS) em 2002-2003 mostrou que, para as 15 capitais analisadas e o Distrito Federal, a cobertura estimada do exame Papanicolaou variou entre $74 \%$ e $93 \%$. Entretanto, o percentual de realização deste exame pelo Sistema Único de Saúde (SUS) variou entre 33\% e 64\% do total dos exames realizados (rede pública e rede privada), o que, em parte, explica o diagnóstico tardio e a manutenção das taxas de mortalidade relacionadas ao câncer do colo do útero, bem como as altas taxas de incidência deste tipo de neoplasia observadas no Brasil ${ }^{(13)}$. Em 2003, no

\begin{tabular}{lllllllll}
\hline \hline Revista Eletrônica & Gestão & \& & Saúde & Vol.04, & №. & 04, & Ano & 2013
\end{tabular}


município de São Paulo, a cobertura do exame foi de aproximadamente $65 \%$ entre as usuárias que o haviam realizado em um período inferior a um ano ${ }^{(14)}$. No município de Igarapava (SP) em 2006, a rede pública municipal realizou uma cobertura de $14,9 \%$ da população feminina na faixa etária de 25 a 59 anos $^{(4)}$. Já em um estudo realizado em Fortaleza (CE), no ano de 2007, observou-se uma cobertura do exame de 11,22\% das usuárias na faixa etária de 25 a 59 $\operatorname{anos}^{(15)}$. No bairro analisado neste estudo, a rede pública obteve uma cobertura de $14,52 \%$ para a população feminina na faixa etária de 25 a 64 anos, resultado semelhante aos encontrados nos estudos de referência, mesmo considerando as diferenças quanto às faixas etárias analisadas.

A maior cobertura realizada pela rede pública no bairro estudado, de $15,46 \%$, ocorreu para a população na faixa etária de 35 a 49 anos. A realização do exame Papanicolaou na população feminina desta faixa etária é importante, considerando-se que esta é a fase na qual ocorre o pico de incidência das lesões precursoras para o desenvolvimento do câncer do colo do útero ${ }^{(6)}$. Ainda assim, esses resultados representam cerca da metade da cobertura mínima, de $30 \%$, que é esperada para os municípios, conforme preconizado pelo $\mathrm{MS}^{(12)}$.

De acordo com a Organização Mundial da Saúde (OMS), conforme citado anteriormente, uma cobertura da população alvo de, no mínimo, $80 \%$ e a garantia de diagnóstico e tratamento adequados dos casos alterados, reduz a incidência do câncer do colo do útero invasivo em 60 a 90\%, em média ${ }^{(8)}$. O Estado do Rio de Janeiro apresentou uma cobertura próxima a 90\%, considerando o total de exames realizados no triênio $2003-2005^{(16)}$. Entretanto, ao analisar a cobertura anual realizada pelo mesmo Estado, no ano de 2009, o resultado foi de $11 \%$, bem abaixo da cobertura de $30 \%$ preconizada pelo MS e aquém da cobertura nacional para o mesmo ano, de $18 \%{ }^{(12)}$. No município ao qual pertence o bairro estudado no presente trabalho, a cobertura do exame, no ano de 2011, para a faixa etária de 25 a 59 anos foi de $10,52 \%$, enquanto a cobertura total para o mesmo ano foi de $6,59 \%$, segundo dados do SISCOLO 2011 e do Censo 2010 do IBGE ${ }^{(17,18)}$. Portanto, observa-se um decréscimo da cobertura quando se analisam os dados nacionais, do Estado do Rio de Janeiro e do município ao qual o bairro estudado pertence. Entretanto, a cobertura total, de 11,25\%, observada no bairro estudado não seguiu essa tendência, mantendo-se acima daquelas observadas no seu município e no seu Estado. Ainda assim os resultados encontrados são preocupantes, visto que estão abaixo do esperado pelo MS

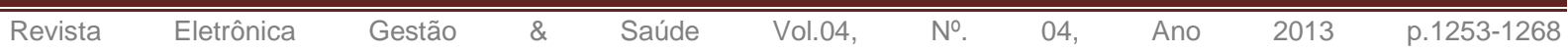


Estudos semelhantes que analisaram as entregas dos resultados do exame Papanicolaou mostraram que um total de 6,30 a 23,98\% das usuárias não compareceram ao serviço de saúde para receber os respectivos resultados ${ }^{(4,10,15)}$. Na Unidade Básica de Saúde (UBS) estudada, 29,90\% das usuárias não compareceram ao serviço de saúde para esta finalidade. Comparando este resultado com aqueles dos estudos de referência, percebe-se uma proporção superior de usuárias que não retornaram para buscar seus resultados. Vale destacar que, em um dos trabalhos de referência obteve-se resultado próximo ao encontrado no presente estudo ${ }^{(15)}$. Entretanto, o intervalo para o recebimento do resultado no trabalho citado variou de dois a onze meses, enquanto que no presente estudo este intervalo variou de cinco a dezessete meses, e, ainda assim, a proporção de usuárias que não compareceram ao serviço de saúde para receber o seu resultado foi quase $6 \%$ maior.

Com relação às alterações encontradas nos resultados dos exames, houve dificuldade de comparação do presente estudo com trabalhos semelhantes, principalmente devido às variações do conceito de resultado alterado que foram empregadas em cada pesquisa. Em um dos trabalhos de referência, encontrou-se uma proporção de $51,6 \%$ de exames dentro dos limites de normalidade, e 47,9\% apresentando alguma alteração, sendo $0,1 \%$ destes compatível com Neoplasia Intraepitelial Cervical (NIC) II e III $^{(4)}$. Já em outro estudo, encontrou-se uma proporção de $94,6 \%$ de exames considerados como sem alterações celulares, incluindo-se nesta proporção os resultados com alterações celulares benignas, e $2,6 \%$ compatíveis com NIC $\mathrm{I}^{(15)}$. Na UBS estudada, $12,76 \%$ dos resultados estavam dentro dos limites de normalidade, enquanto $78,66 \%$ apresentaram alguma alteração, sendo $0,57 \%$ dos resultados compatíveis com Lesão Intraepitelial escamosa de baixo grau (LSIL) equivalente a NIC I. No presente estudo não houve casos de NIC II e III. Somando-se os resultados normais com os resultados que apresentaram alterações celulares benignas, obtevese $90,28 \%$ de resultados sem alterações celulares importantes, semelhante ao encontrado em um dos estudos de referência ${ }^{(15)}$.

\section{CONCLUSÃO}

\begin{tabular}{|c|c|c|c|c|c|}
\hline Revista & Eletrônica & Gestãc & & & \\
\hline
\end{tabular}


O exame de Papanicolaou é um método de rastreamento do câncer do colo do útero, que permite a detecção precoce das lesões pré-neoplásicas, bem como dos agentes de processos inflamatórios que causam desconforto. A Unidade Básica de Saúde (UBS) estudada realizou em 2011, 525 coletas do exame Papanicolaou, apresentando uma cobertura de 5,40\% da população feminina do bairro, sendo que a cobertura total do bairro, considerando também os 586 exames realizados pelas Unidades de Saúde da Família (USF), foi de 11,42\%. A cobertura do exame para a faixa etária de 25 a 64 anos, foi de 14,52\% no mesmo bairro, configurando uma cobertura inferior à cobertura de $30 \%$ preconizada pelo Ministério da Saúde (MS).

Um total de 29,90\% das usuárias não compareceu à UBS a fim de receber o resultado do seu exame. Esta proporção mostrou-se superior àquelas encontradas em estudos de referência. Observa-se com isso, uma deficiência nas ações de busca ativa e de orientação das usuárias acerca do seu acompanhamento.

Em relação aos resultados dos exames, $12,76 \%$ apresentaram resultados normais, enquanto $78,66 \%$ apresentaram alterações, com predomínio de alterações celulares benignas. Apenas $0,57 \%$ do total de resultados foram compatíveis com Lesão Intraepitelial escamosa de baixo grau (LSIL), alteração esta que regride espontaneamente na quase totalidade dos casos.

Também foram constatadas falhas nos registros de informações sobre a entrega dos resultados em $15,24 \%$ dos casos, sobre a presença de alterações nos exames em $8,57 \%$ dos casos e sobre a conduta médica por anotação ilegível $(6,25 \%)$, por ausência de anotação no prontuário $(8,68 \%)$ ou por prontuários não encontrados $(13,54 \%)$. Isto mostra claramente a necessidade de se promover a conscientização dos profissionais de saúde da UBS em relação ao preenchimento adequado de todo e qualquer registro de informação, para que assim, possa haver preciso reconhecimento da realidade da população usuária. Neste sentido, verificou-se também a necessidade de se classificar os resultados dos exames de acordo com o protocolo do MS de Nomenclatura Brasileira para Laudos Cervicais e Condutas Preconizadas, visando permitir uma análise mais apurada da gravidade das lesões e facilitando comparações com outros estudos. Tais esforços poderiam trazer impactos positivos na melhoria da cobertura dos exames Papanicolaou e na adesão das usuárias ao tratamento, contribuindo para a redução da incidência de câncer do colo do útero.

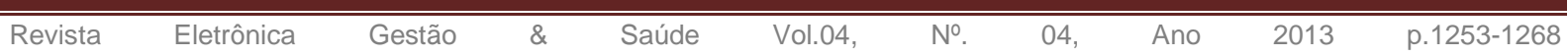


Por fim, torna-se evidente a necessidade de se investigar as razões que levam as mulheres a não retornarem para buscar os resultados. A adesão da paciente, principalmente em casos de resultados com alterações, é necessária para que o tratamento adequado seja promovido, e o seguimento da atenção à saúde desta usuária seja plenamente realizado, permitindo a redução da incidência desse tipo de neoplasia. Também é importante conhecer a cobertura total do exame Papanicolaou (rede pública e rede privada) na população feminina estudada.

\section{LIMITAÇÕES}

No decorrer deste trabalho de pesquisa foram identificadas algumas falhas no processo de registro de informações na UBS, o que dificultou a obtenção de dados estatísticos precisos. Essas falhas incluem a divergência de informações entre o Livro Controle, a Planilha Controle e os Prontuários; a organização precária do Arquivo de Exames; o preenchimento deficiente do Livro Controle; o preenchimento deficiente e anotações ilegíveis nos Prontuários; Prontuários não encontrados; e por fim, os mais de 16 tipos de alterações consideradas, que foram registradas de forma incompatível com o protocolo do Ministério da Saúde de Nomenclatura Brasileira para Laudos Cervicais e Condutas Preconizadas.

\section{REFERÊNCIAS BIBLIOGRÁFICAS}

1) Ministério da Saúde (BR), Instituto Nacional do Câncer. Periodicidade de realização do exame preventivo do câncer do colo do útero. Disponível em: www.saude.pr.gov.br/cancer/colo_ utero/periodo.html/ Acesso em 16 de outubro de 2012.

2) Instituto Nacional do Câncer (INCA). Disponível em: http://www2.inca.gov.br/wps/wcm/ connect/tiposdecancer/site/home+/c olo_utero/definicao/ Acesso em $29 / 11 / 2012$

3) Muller DK, Dias da Costa JS, Lus AMH, Olinto MTA. Cobertura do exame citopatológico do colo do útero na cidade de São Leopoldo, Rio Grande do Sul, Brasil. Cad Saúde Pública. 2008; 24(11):251120.

4) Soares MBO, Silva SR. Análise de um programa municipal de 
prevenção do câncer cérvicouterino. Rev Bras Enferm, Brasília. 2010; 63(2): 177-82.

5) Instituto Nacional do Câncer (BR). Diretrizes Brasileiras para o Rastreamento do Câncer do Colo do Útero. Rio de Janeiro. INCA, 2011.

6) Ministério da Saúde (BR), Instituto Nacional do Câncer, Secretaria de Estado da Saúde (FOSP). Coleta do Papanicolau e ensino do autoexame da mama manual de procedimentos técnicos e administrativos. $2 \mathrm{a}$ ed. São Paulo. Imprensa Oficial; 2004.

7) World Health Organization. Cancer Control. Knowledge into ation. WHO guide for efective programmes. Switzerland: WHO, 2007.

8) World Health Organization. National cancer control programmes: policies and managerial guidelines. 2.ed. Geneva: WHO, 2002.

9) Pinho AA. Fatores associados à realização do Teste de Papanicolau entre mulheres em idade reprodutiva no município de São Paulo [dissertação]. São Paulo: Mestrado em saúde MaternoInfantil, Universidade de São Paulo; 2002.
10) Greenwood AS, Machado MFAS, Sampaio NMV. Motivos que levam mulheres a não retornarem para receber o resultado de exame Papanicolau. Rev. Latino-Am. Enfermagem. 2006; 14(4): 503-9.

11) Travassos C, Martins M. Uma revisão sobre os conceitos de acesso e utilização de serviços de saúde. Cad. Saúde Pública. 2004; 20(2): 190-198.

12) Ministério da Saúde (BR). Orientações acerca dos Indicadores de Monitoramento -Avaliação do Pacto pela Saúde, nos Componentes pela Vida e de Gestão para o Biênio 2010 - 2011.

13) Ministério da Saúde/INCA. Nomenclatura Brasileira para Laudos Cervicais e Condutas Preconizadas, Recomendações para profissionais de saúde. 2006.

14) Pinho AA, França I Junior, Schraiber LB, D'Oliveira AFPL. Cobertura e motivos para a realização ou não do teste de Papanicolau no Município de São Paulo. Cad Saúde Pública. 2003; 19(2):303-13.

15) Vasconcelos CTM, Neto JAV, Castelo ARP, Medeiros FC, Pinheiro AKB. Análise da cobertura e dos exames 
colpocitológicos não retirados de uma Unidade Básica de Saúde. Rev Esc Enferm USP. 2010; 44(2): 32430.

16) Szwarcwald CL, Mendonça MHM, Andrade CLT. Indicadores de atenção básica em quatro municípios do Estado do Rio de Janeiro, 2005: resultados de inquérito domiciliar de base populacional. Cien. Saude Colet. 2006.

17) Instituto Brasileiro de Geografia e Estatística. 2010. Disponível em: www.sidra.ibge.gov.br/ Acesso em 07 de outubro de 2012.

18) Sistema de Informação do Câncer de Colo do Útero. 2011. Disponível em: siscam.datasus.gov.br/ Acesso em 14 de outubro de 2012. 